Int. J. Dev. Biol. 58: 57-64 (2014)

doi: $10.1387 / \mathrm{ijdb} .130023 \mathrm{sy}$

\title{
Developmental expression of chicken FOXN1 and putative target genes during feather development
}

\author{
DIANA K. DARNELL*,1, LI S. ZHANG ${ }^{1}$, SRIDHAR HANNENHALLI ${ }^{2}$ and SERGEY Y. YAKLICHKIN ${ }^{*, 3}$ \\ ${ }^{1}$ Department of Cellular and Molecular Medicine, University of Arizona, Tucson, AZ, \\ ${ }^{2}$ Center for Bioinformatics and Computational Biology, University of Maryland, College Park, MD and \\ ${ }^{3}$ Department of Molecular and Cellular Biology, Baylor College of Medicine, Houston TX, USA
}

\begin{abstract}
FOXN1 is a member of the forkhead box family of transcription factors. FOXN1 is crucial for hair outgrowth and thymus differentiation in mammals. Unlike the thymus, which is found in all amniotes, hair is an epidermal appendage that arose after the last shared common ancestor between mammals and birds, and hair and feathers differ markedly in their differentiation and gene expression. Here, we show that FOXN1 is expressed in embryonic chicken feathers, nails and thymus, demonstrating an evolutionary conservation that goes beyond obvious homology. At embryonic day (ED) 12, FOXN1 is expressed in some feather buds and at ED13 expression extends along the length of the feather filament. At ED14 FOXN1 mRNA is restricted to the proximal feather filament and is not detectable in distal feather shafts. At the base of the feather, FOXN1 is expressed in the epithelium of the feather sheath and distal barb and marginal plate, whereas in the midsection FOXN1 transcripts are mainly detected in the barb plates of the feather filament. FOXN1 is also expressed in claws; however, no expression was detected in skin or scales. Despite expression of FOXN1 in developing feathers, examination of chick homologs of five putative mammalian FOXN1 target genes shows that, while these genes are expressed in feathers, there is little similarity to the FOXN1 expression pattern, suggesting that some gene regulatory networks may have diverged during evolution of epidermal appendages.
\end{abstract}

KEY WORDS: chick, forkhead, WHN, HFH11, thymus, nudee

The forkhead family transcription factor FOXN1 (formerly called Winged helix transcription factor nude, Whn (Meier et al., 1999) and HNF-3/forkhead homolog 11, Hfh11 (Segre et al., 1995) is essential for hair and thymus development in mammals. Hair production is complex and cyclic, involving many transcription factors and their targets (Lee and Tumbar, 2009; Lin et al., 2009). In the murine hair follicle FOXN1 exhibits a dynamic expression pattern that is dependent on the stage of the hair cycle. FOXN1 appears to be strongly expressed in the anagen or growth phase and its expression decreases by the telogen or quiescent phase. In the mature hair follicle FOXN1 is expressed in the hair shaft and the inner root sheath (Lee at al., 1999). In the hair shaft, FOXN1 is expressed in the cortex, which is consistent with absence of the cortex in Nude (FOXN1 $1^{n u} /$ nu $)$ mice. Nude mice develop normal hair follicles and produce hair shafts, but the cortex fails to form most keratins so the hair does not extend. The absence of visible hair is due to a coiling of hair shafts in the hair canal resulting in a failure to penetrate the epidermal layer of the skin or breaking off at the surface (Mecklenburg et al., 2001, 2004, 2005). Similarly, humans with FOXN1 mutation have congenital alopecia (hair loss), nail dystrophy and immunodeficiency (Frank et al., 1999, Pignata et al., 2009).

Although FOXN1 function is well studied in mammals, and additional work has been done on its thymic expression in zebrafish (Ma et al., 2012), frog (Lee et al., 2013) and birds (Neves et al., 2012), nothing is known about FOXN1 expression in the integumentary organs in non-mammalian vertebrates. Birds, like mammals, have developed evolutionarily novel integumentary appendages, feathers, which are composed of keratins. But as hair and feathers

Abbreviations used in this paper: ED, embryonic day; FOX, forkhead box transcription factor.

\footnotetext{
*Address correspondence to: Diana K. Darnell. Department of Cellular and Molecular Medicine, 1656 E. Mabel Street - MRB324, Tucson, AZ 85724, USA.

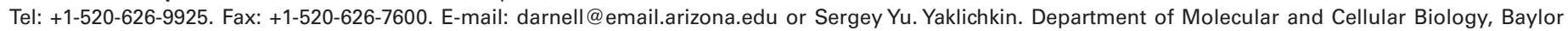
College of Medicine, One Baylor Plaza N620, Houston, Texas 77030, USA. E-mail: sergey.yaklichkin@bcm.edu
}

Supplementary Material (table) for this paper is available at: http://dx.doi.org/10.1387/ijdb.130023sy

Accepted: 9 January 2014. Final, author-corrected PDF published online: 17 February 2014.

ISSN: Online 1696-3547, Print 0214-6282 
A FoxN1_zebrafinch

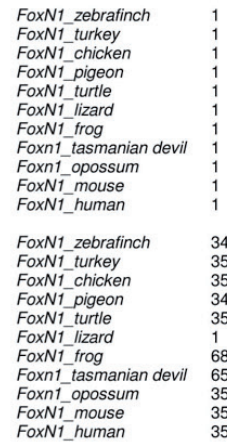

FoxN1_zebrafinch

FoxN1_turkey

FoxN1_chicken

FoxN1_pigeon

FoxN-1izard

Foxnittrag asmanian devil
Foxn_topossum

FoxN1_mouse
FoxN1_human

FoxN1_zebratinch

FoxN1_turkey
FoxN1_chicken

FoxN1 pigeon

FoxN1-1izard

FoxN1_trog

Foxn1-tasmanian devil

FoxN1 mouse

FoxN1_zebratinch

FoxN1_turkey

FoxN1-chicken

FoxN1_pigeon
FoxN1_turtle

FoxN1_lizard
FoxN1 frog

Foxn1 tasmanian devil

Foxnt_opossum

FoxN1_human

FoxN1_zebrafinch

FoxN1_turkey
FoxN1_chicken

FoxN1-chicken

FoxN1_turtle

FoxN-1 lizard
FoxN1 frog

Foxn1_tasmanian devil

Foxnt opossum

FoxN1_human

FoxN1_zebrafinch

FoxN1_turkey

FoxN1_chicken

FoxN1_pigen

FoxN1turte

FoxN1_lizard
FoxN1_trog

Foxn1_tasmanian devi

Foxnt_opossum

FoxN1_human

FoxN1_zebratinch

FoxN1 turkey
FoxN1 chicken

FoxN1_ phigeon

FoxN1 turtle

FoxN1_lizard

FoxNi_trog

Foxn1_tasmanian devil

Foxnt_opossum
FoxN1_mouse

FoxN1_human

FoxN1_zebrafinch

FoxN1 turkey
FoxN1 chicken

FoxN1-pigeon

FoxN1_turtle

FoxN1_lizard
FoxN1_trog

Foxn1_tasmanian dev

Foxnt opossum
FoxN1 mouse

FoxN1_human

FoxN1_zebrafinch

FoxN1_turkey
FoxN1_chicken

FoxN1-chicken
FoxN_pigeon

FoxN1-turtle

FoxN-1izard

FoxN1_tasmanian devit

Foxn1_opossum

FoxN1_mouse
FoxN1_human

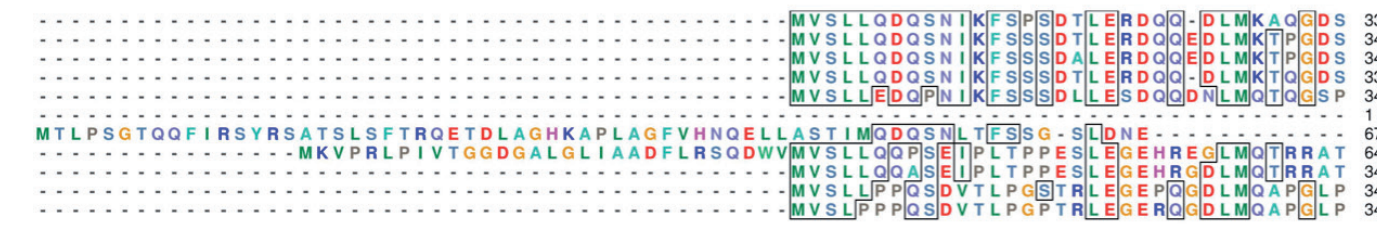

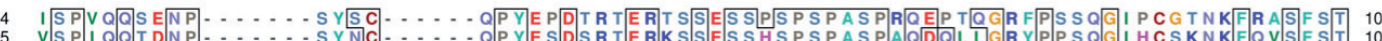

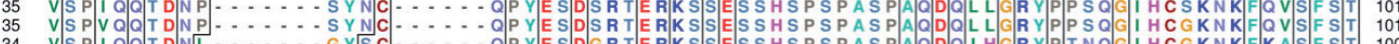

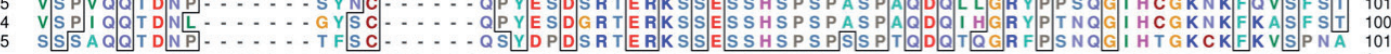

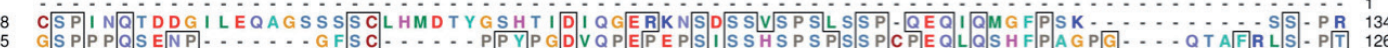

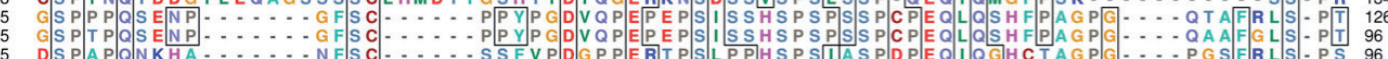

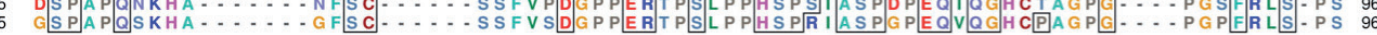

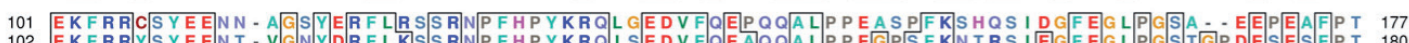

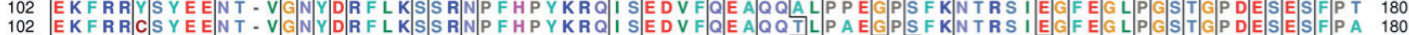

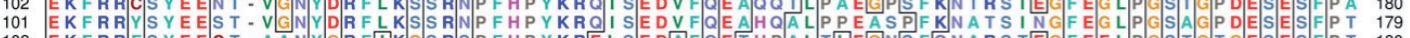

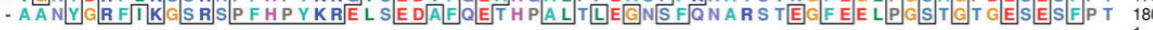

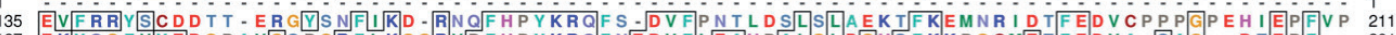

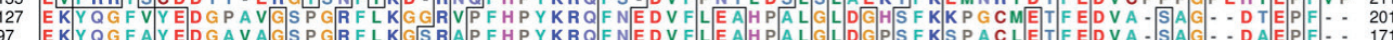

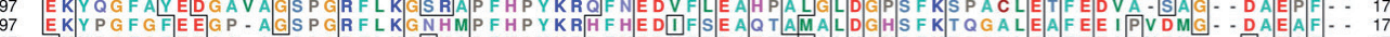

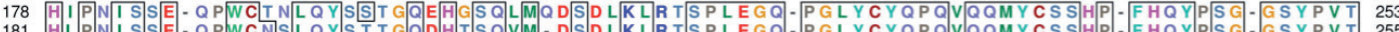

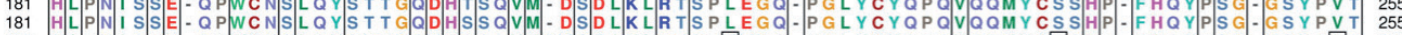

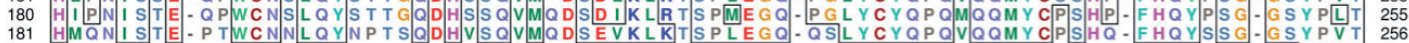

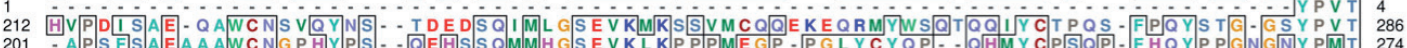
201
171

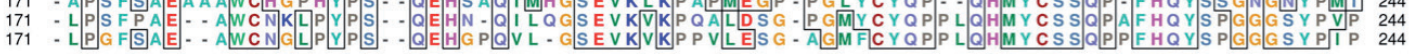
254 YIASSHYPYQRIAPQSSSOESQQPLFPKPIYSYSILIFMALKNSKTGSLPVSEIYNFMTEHFPYFKTAPDGWKNSVRHNLS 33 256 Y ITSSHYPYQRIAPQSTOESQQPLFPKPIYSYSILIFMALKNSKTGSLPVSEIYNFMTEHFPYFKTAPDGWKNSVRHNLS 335 256
256 ITSSHYPYORIAPQSTOESQQPLFPKPIYSYSILIFMALKNSKTGSLPVSEIYNFMTEHFPYFKTAPDGWKNSVRHNLS 335 257
257
$Y$ IISTSHYPYQRIAPQGPPE HHQPLFPKP I YSYSILIFMALENSKTGSLPVSEI YNFMTEHFPYFKTAPDGWKNSVRHNLS 287 Y IST SHYPYQRIAPOASMEAAQPLVPKPIYSYS ILIFMALKNSKTGSLPVSE I YNFMTEHFPYFKTAPDGWKNSVRHNLS 360 275
245
$Y$

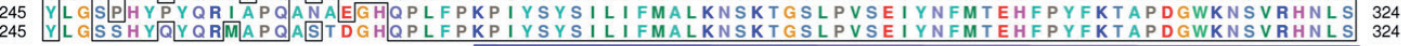
334 LNKCFEKVENKSGNSSRKGCLWALNPAKIDKMOEELOKWKRKDPVAVRKSMAKPEELDTLIGDKS EKLLRSSLASA-S PTT 412

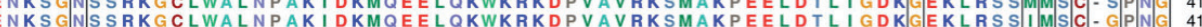
336 LNKCFEKVENKSGNSSRKGCLWALNPAKI DKMQEELQKWKRKDPVAVRKSMAKPEELDTLIGDKSEKLRSSVVSC.SPTG 414 37 LNKCFEKVENKSGGSSRKGCLWALNPAKI DKMQEELOKWKRKDPVAVRKSMAKPEELDTLIGEKAEKLRSSAMS C. NPTG 415

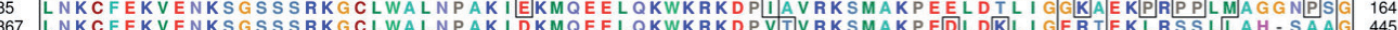
367 LNKCFEKVENKSGSSSRKGCLWALNPAKI DKMQEELOKWKRKDPVTVRKSMAKPEDLDEL I GERTEKLRSSLLAH-SAAG 445

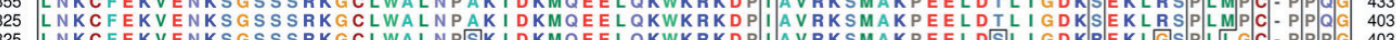

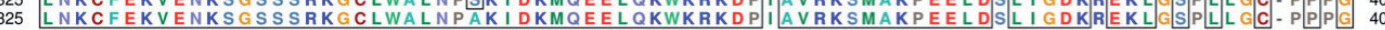

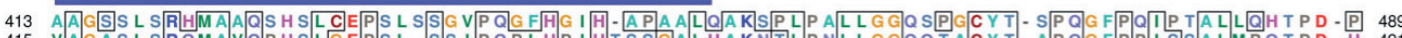

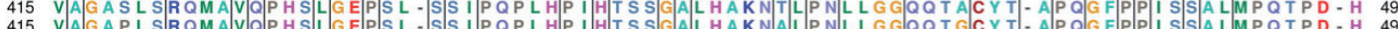
415 $\begin{array}{ll} & \end{array}$ 165 VGS - . BQD V P L 作

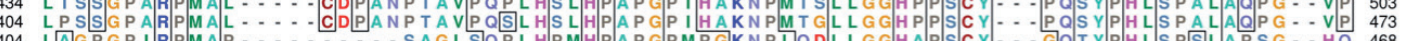

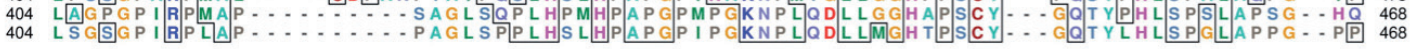

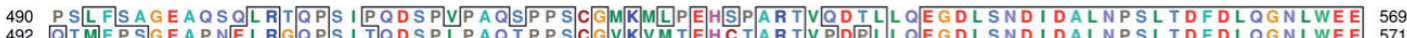
492 O O TMEP S A A N

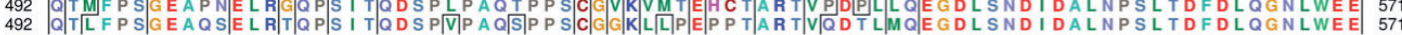

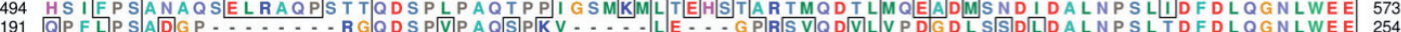

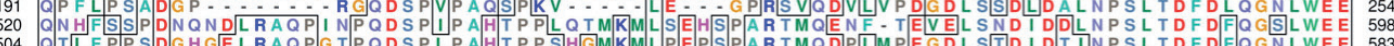

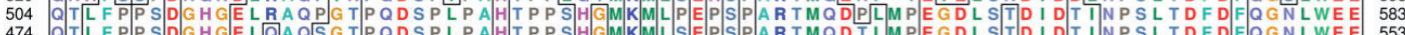
469 OPLFPOPDG

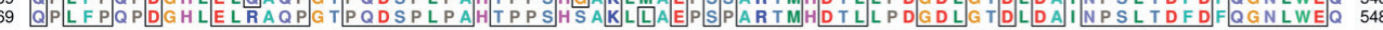

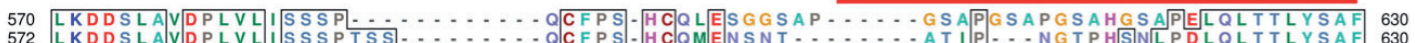

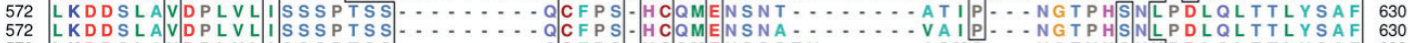
574 LKDDSLAVIPLVLLSTSP IS

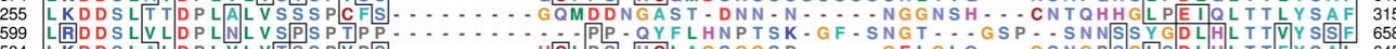
584

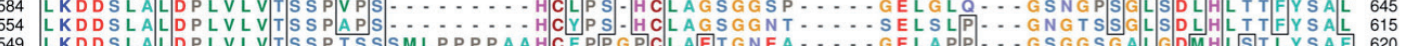

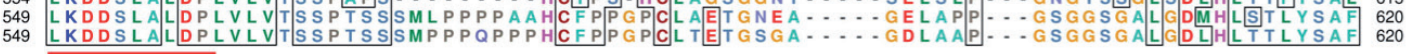

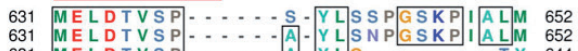

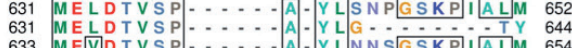
641 MELDTVST......... A

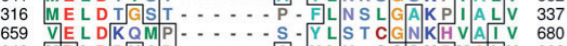
646 Min

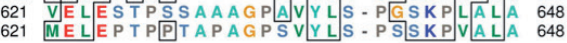




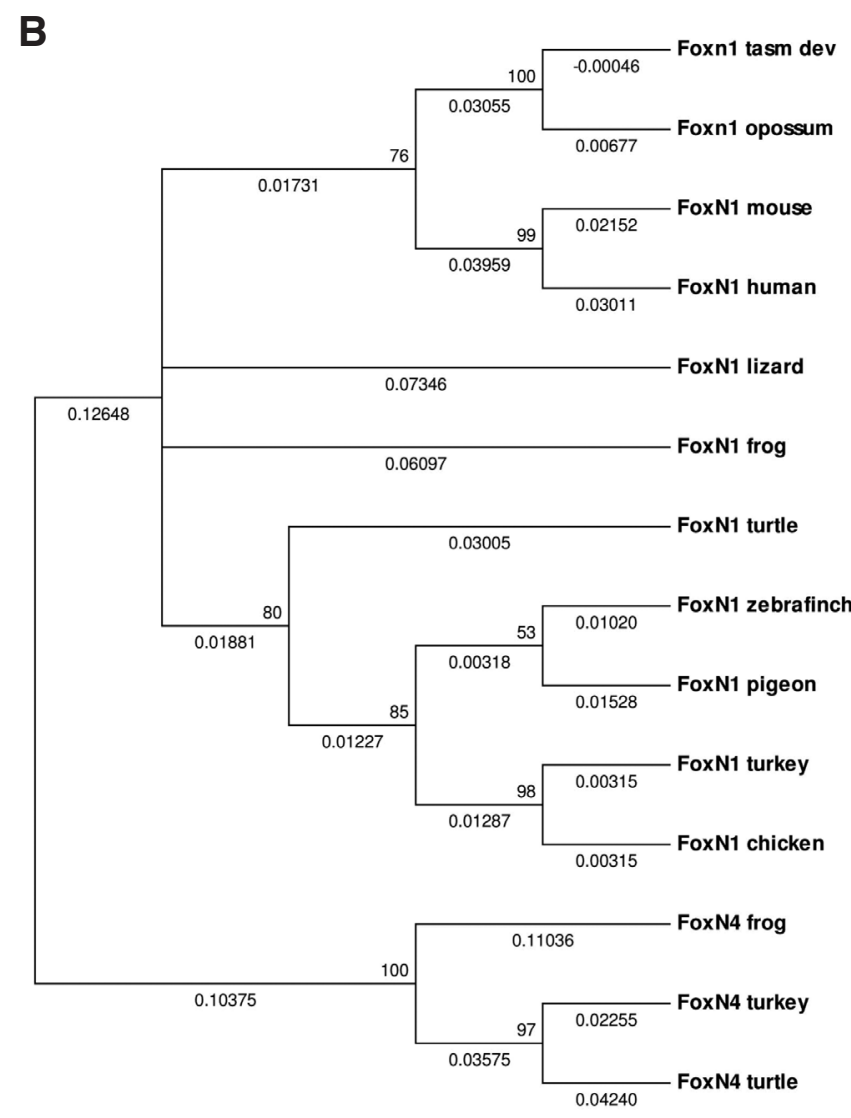

Fig. 1 (left and above). Comparison of amino acid sequences of avian FOXN1 with orthologous proteins and phylogenetic tree of FOXN1/ N4. (A) Predicted amino acid sequences of avian FOXN1 proteins aligned with orthologous protein sequences using T-coffee algorithm. The conserved forkhead DNA-binding (Kaestner et al., 2000) and transcriptional activation domains (Schüddekopf et al., 1996) are underlined in blue and red, respectively. Similar amino acid residues are shaded, threshold $70 \%$. (B) A phylogenetic tree of the FOXN1 proteins, with FoxN4 protein sequences used as an outgroup. A neighbor-joining method was used to construct the tree topology based on the forkhead DNA-binding domain.

arose after the last shared common ancestor between mammals and birds in the late Carboniferous period, it is not surprising that proteins in these two lineages have functionally diverged (Furlong, 2005). For example, hair is characterized by a preponderance of soft alpha keratin (Eckhart et al., 2008), whereas feathers are characterized predominantly by the harder beta keratins (Glenn et al., 2008). Alpha keratins are found in the feather sheath and barb ridges but are rapidly overwhelmed by deposition of beta keratins (Alibardi, 2013). Since one function of transcription factor FOXN1 in mice is the activation of alpha keratins 2-6 (Schlake et al., 2000; Mecklenburg et al., 2004) and birds appear primarily to use beta keratin proteins in feathers, it has not been tested whether feathers express FOXN1. We previously showed that another Forkhead transcription factor, FOXE1, is transcribed in the feather filament (Yaklichkin et al., 2011), and its mammalian ortholog is implicated in hair morphogenesis (Brancaccio et al., 2004). This suggests the hypothesis that FOXN1 and homologs of its mammalian regulatory targets might also be expressed in developing feathers.

In this report we show that, whereas FOXN1 and its putative regulatory targets are indeed expressed in developing feathers, some of the gene regulatory pathways downstream of FOXN1 in epidermal appendage growth appear to have diverged since the division between mammal and bird ancestors about 310 million years ago (Kumar and Hedges, 1998).

\section{Results}

\section{Phylogenetic and sequence analysis of avian FOXN1 sequences}

The chick FOXN1 gene encodes a 653 amino acid protein with a molecular weight of $71,957 \mathrm{Da}$. The chick FOXN1 gene shares synteny with mammalian FOXN1, thus providing unambiguous evidence for orthology. Of non-avian orthologs, chick FOXN1 protein sequence overall shares the highest sequence identity, $75 \%$, with turtle and lizard FOXN1, although the lizard protein sequence is incomplete (Fig. 1A). For comparison, Chick FOXN1 protein shares $59 \%$ and $53-55 \%$ sequence identity with opossum and placental FOXN1 (Fig. 1A). To determine the evolutionary relatedness of avian FOXN1 gene to orthologs, a phylogenetic analysis was performed using the Neighbor-joining method (Kumar et al., 2004). The phylogenetic tree shows that avian FOXN1 genes are clustered within the FOXN1 group and the closest phylogenetic relatedness is observed to the reptilian and amphibian FOXN1 genes, which is supported by the high bootstrap values (Fig. 1B).

\section{Expression of chick FOXN1 gene in developing thymus}

We performed whole mount in situ hybridization analysis to examine FOXN1 expression during chick thymus development from stages ED1-16. No expression was detected from ED1-4.5 (not shown). In chicken, thymus is derived from the third and/or fourth pharyngeal pouch endoderm at stage ED5. At about ED9 thymus primordia detach from the pharynx and migrate to their mature locations in the neck, where they form bilateral multi-lobed glands near the jugular vein. We found that FOXN1 mRNA was strongly expressed bilaterally in ED9 thymus (Fig. 2A). A section through the ED9 thymus primordia shows that FOXN1 labeling is stronger in the prospective cortex with uniform lower intensity label in the medulla (Fig. 2B). This is consistent with the proposed function of mouse FOXN1 in regulation of keratin gene expression, which is present in medullary and cortical thymus epithelial cells during early thymus development (Rodewald, 2008; Nowell et al., 2011). As chick embryonic development progresses (stage ED13) the thymus lobes migrate laterally and expand; expression of FOXN1 persisted (Fig. 2C-D). A section through the thymus at this stage shows increased intensity of FOXN1 mRNA labeling in the cortex (Fig. 2E). This is consistent with the observation in mouse that a FOXN1 mutant phenotype is more severe in the cortical compartment, although both cortex and medulla are affected (Nowell et al., 2011); and expands on the published FOXN1 expression at older stages in chick: (ED18; Neves et al., 2012).

\section{Expression of chick FOXN1 gene in developing feathers}

Feathers are unquestionably the most complex vertebrate epidermal appendages and the specifics of their embryology are well described (e.g., Yu et al., 2002, 2004; Alibardi, 2009, 2013). In short, a feather placode in the epidermis with an underlying dermal condensation sprouts into a feather filament with a dermal pulp separated from the epidermal sheath by the pulp epithelium. Barb 

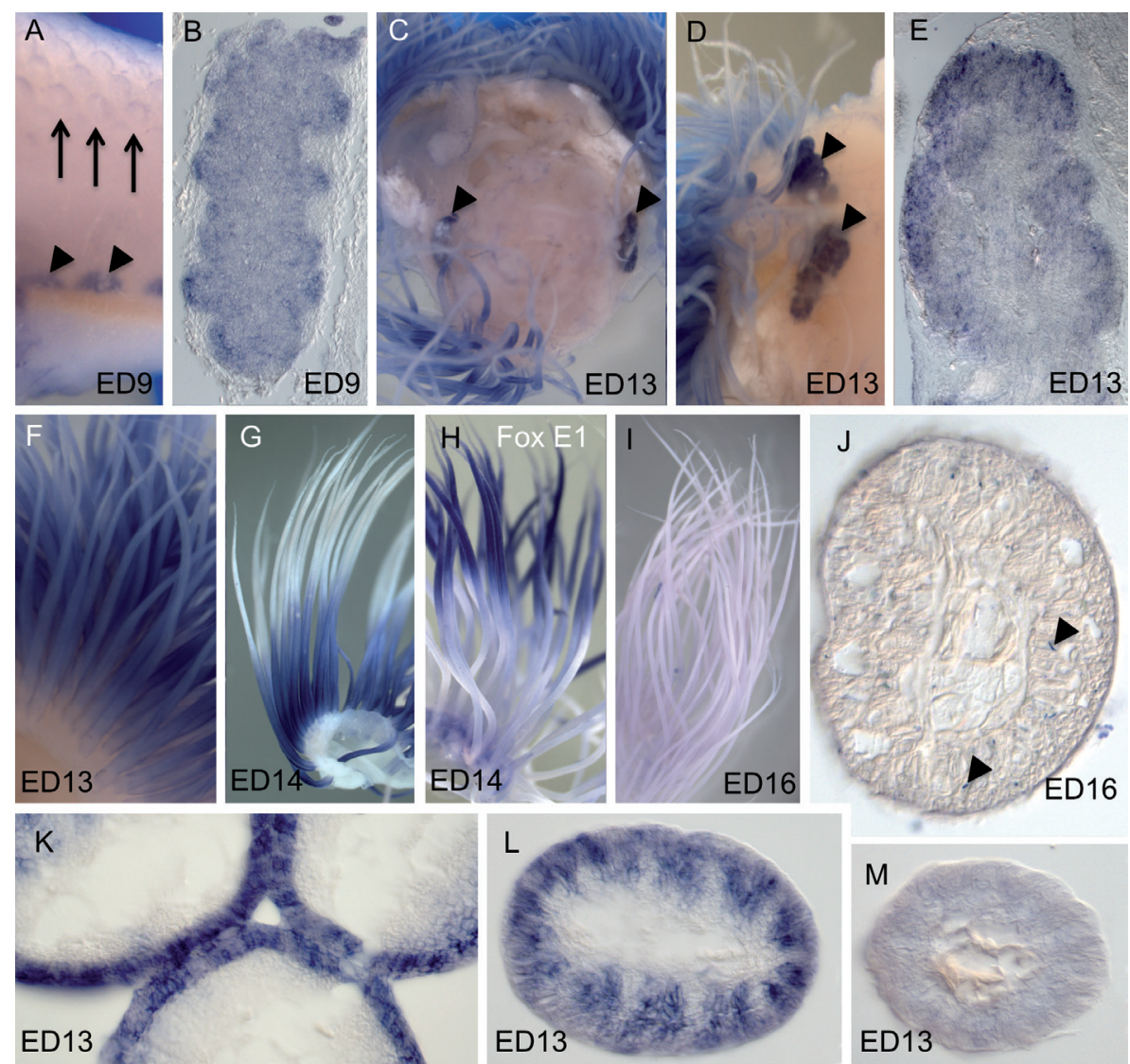

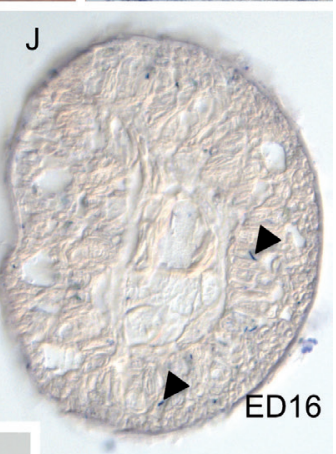

M

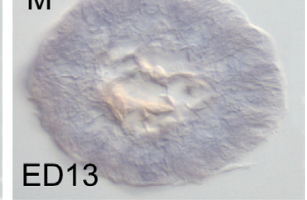

Fig. 2. Expression of chick FOXN1 in developing thymus and feathers. (A) FOXN1 expression (arrowheads) in ED9 chick neck showing one side of bilateral, lobular expression in the thymus glands. Early feather buds (arrows) and skin show no expression. (B) A section through the thymus lobe in panel A showing expression throughout the thymus with increased intensity in the cortex. (C) Bilateral FOXN1 expression (arrowheads) in ED13 chick neck looking down from above after decapitation. Expression in feathers filaments can be seen peripherally. (D) Another ED13 embryonic neck from the side after removal of the skin showing two lobes of the thymus (arrowheads). (E) A section through ED13 thymus shows expression varies in intensity with stronger expression in the cortex. (F) FOXN1 in situ hybridization of feather filaments from ED13 showing label along the entire length of the shaft, but less intense at the base and tip. (G) ByED14, FOXN1 mRNA expression restricted to the proximal shaft, whereas in comparison with skin from a similarly-staged embryo, FOXE1 mRNA expression $(\mathbf{H})$ is limited to the distal shaft of the feather. (I) At a slightly older stage the expression of FOXN1 mRNA in the feather was absent. Trapped precipitate ( $\mathbf{J}$-arrowheads) that can be seen in cross section of (I) indicates color reactants were able to penetrate. A transverse section near the base (K), midpoint (L) or tip of the shaft (M) of feathers similar to those shown in panel (F) indicates a transition in both morphology of the feather and label. ridges begin to form in one side of the epidermis and are displaced laterally to create two vertical half-spirals or arcs between the base and their distal merger into the feather stem or rachis on the opposite side (downy feathers have no rachis). Each barb ridge appears as an arch in cross section with paired stacks of barb plates at its base and a growth zone at its apex, all surrounded by marginal cells. At maturity, a medullary core forms in each barb, the feather sheath is lost and the barbs separate.

Because FOXN1 is important for normal hair development in mammals, we used in situ hybridization to examine expression of FOXN1 in developing chicken feathers. FOXN1 expression was at, or barely above, background levels in initial feather buds at ED9 (Fig. 2A), but strong transient expression was observed in feather filaments at stages ED13-16 (Fig. 2 F-G, I-M). Strong expression of FOXN1 mRNA in the feather filament was detected nearly from base to apex of the feather at stage ED13 (Fig. 2F). Weaker FOXN1 label was observed in the most proximal part of the feather filament and the most distal tip. This may indicate the beginning of the maturation of the distal region of the filament. By stage ED14 FOXN1 transcripts become restricted to the proximal feather filament (Fig. 2G), which is a less differentiated and morphologically more active portion of the feather filament (Haake et al., 1984). To verify that the failure to hybridize in the distal filament was not due to exclusion of the color reaction or another artifact, in situhybridizations were performed on comparable feather filaments using a probe for another transcription factor, FOXE1 (Fig. $2 \mathrm{H}$; Yaklichkin et al., 2011). FOXE1 showed a complementary expression pattern to that of FOXN1 in proximal/distal distribution at this stage, indicating that both proximal and distal filaments were able to label. By stage ED16, FOXN1 mRNA was no longer detected in the feather filament (Fig. 2I, J), which can be explained by either down-regulation of FOXN1 expression in a differentiated feather filament or increased keratinization in the feather sheath, which may block FOXN1 probe penetration at this stage. Small flecks of trapped color precipitate (artifact, arrowheads Fig. 2J) indicate that probe and labeling solution were able to penetrate.

To determine specific localization of FOXN1 mRNA within the labeled feather filament, feathers were paraffin sectioned transversely. FOXN1 expression was evident in the proximal section of the feather filament at ED12-13 in a tight peripheral band with alternating intensity (Fig. $2 \mathrm{~K}$ ) the 'ring sheath cells' (Haake et al., 1984). At the midpoint of the developing filament, FOXN1 mRNA was expressed within the barb plates (Fig. 2L). At the feather tips, expression was much diminished (Fig. 2M). Thus, our data suggest that FOXN1 may play a role in regulation of differentiation programs or morphogenesis of the feather filament.

\section{Expression of FOXN1 and its putative target genes in cutaneous appendages}

Putative downstream target genes of FOXN1 have been identified in mouse thymocytes (Nowell et al., 2011) and human embryonic stem cells (hESNet). In addition to regulating keratins (e.g., Mecklenburg et al., 2004), the regulation of MHC Class II genes, BRD2 (Bromodomain-containing gene 2), CTSL (Cathepsin L, a lysosomal protease), $C D 40$ (TNF-related receptor), and $D L L 4$ (delta-like gene 4) was positively correlated with FOXN1 dosage (Nowell et al., 2011; hESnet). To determine if these components represent a gene regulatory network that is conserved in other FOXN1-expressing 
tissues, we used in situ hybridization to evaluate the expression of chick homologs of these genes in feathers and other epidermal appendages. Chick BLA2 represented the MHC Class II genes, while other chick gene symbols are similar to mouse. Comparisons of expression in whole mount and feather section are shown in Figures 3 and 4 . Our section photos were taken near the base of the feather filament where barb ridges are first forming on one side (Fig. 4. ED12), or at the proximodistal level corresponding to the densest label for the majority of genes (ED14, ED17). While each of the potential targets exhibited expression in feather filaments and overlapped in expressing regions with FOXN1, none of the patterns were highly similar to FOXN1 in either whole mounts (Fig. 3 ) or cross sections of feathers (Fig. 4). FOXN1 expression was not detected as extensively in wing feathers or skin at ED12 (Fig. $3 \mathrm{~A}$ ), whereas the other five genes were strongly expressed (Fig. 3 B-F). At ED14, four of the putative target genes were expressed in

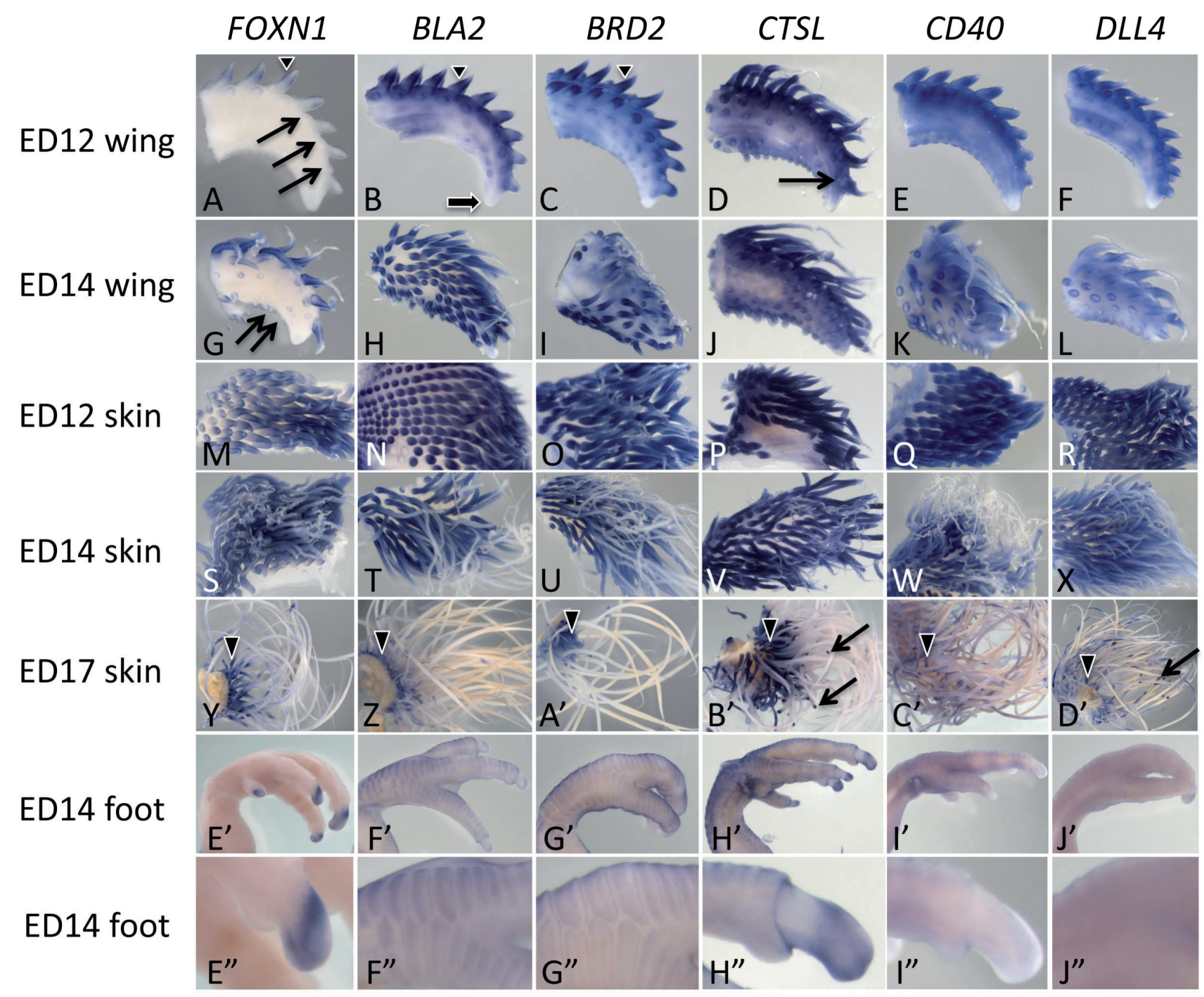

Fig. 3. Expression of FOXN1 and putative FOXN1 regulatory targets in embryonic day 12, 14 and 17 epidermal appendages. Photo columns are labeled with the gene name for the probe, rows indicate the days of incubation and tissue or region shown. All in situ probes labeled in feathers on embryonic day 12 (A-F, M-R), 14 (G-L, S-X) and 17 (Y-D'). (A-F) Embryonic day 12 wing tips. (A) FOXN1 was expressed in some feather buds with stronger expression at the midpoint of the shaft (arrowhead) and lower expression at the base and tip. Some smaller buds (arrows) and the skin did not express. (B-F) The other five genes were also expressed more intensely in the mid-shaft (arrowhead) than at the base or tip, but also in skin and all feather buds. The distal wing tip had less expression for all genes tested (arrow in B). In wing tips at day 14, some feather buds still had no detectable FOXN1 (G, arrows) but most feather buds showed stronger gene expression (G-L). At day 14 of incubation, wing skin had no detectable expression of FOXN1 (G) and BLA2 (H), but expressed other genes (I-L). Day 12 (M-R) and 14 (S-X) feathers from the back of the neck expressed all six genes similarly at this gross level. By day 17, feathers all showed probe binding proximally (all probes, arrowheads, Y-D'). Where the sheath had been bent or cut (e.g., arrows in B', D'), damaged tissue stained. No skin label was detected. ED14 foot: An evaluation of foot scales and nails, two other epidermal appendages. (E') FOXN1 was expressed solely in the nails. Two genes were expressed in the scales (F', G'). CTSL ( $\left.\mathbf{H}^{\prime}\right)$ was expressed in both scales

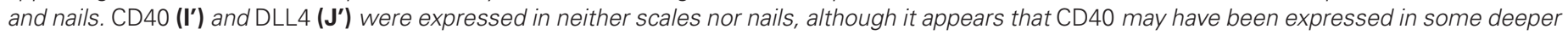
tissue (I'). Final row shows close ups of ( $\left.E^{\prime}-J J^{\prime}\right)$. 


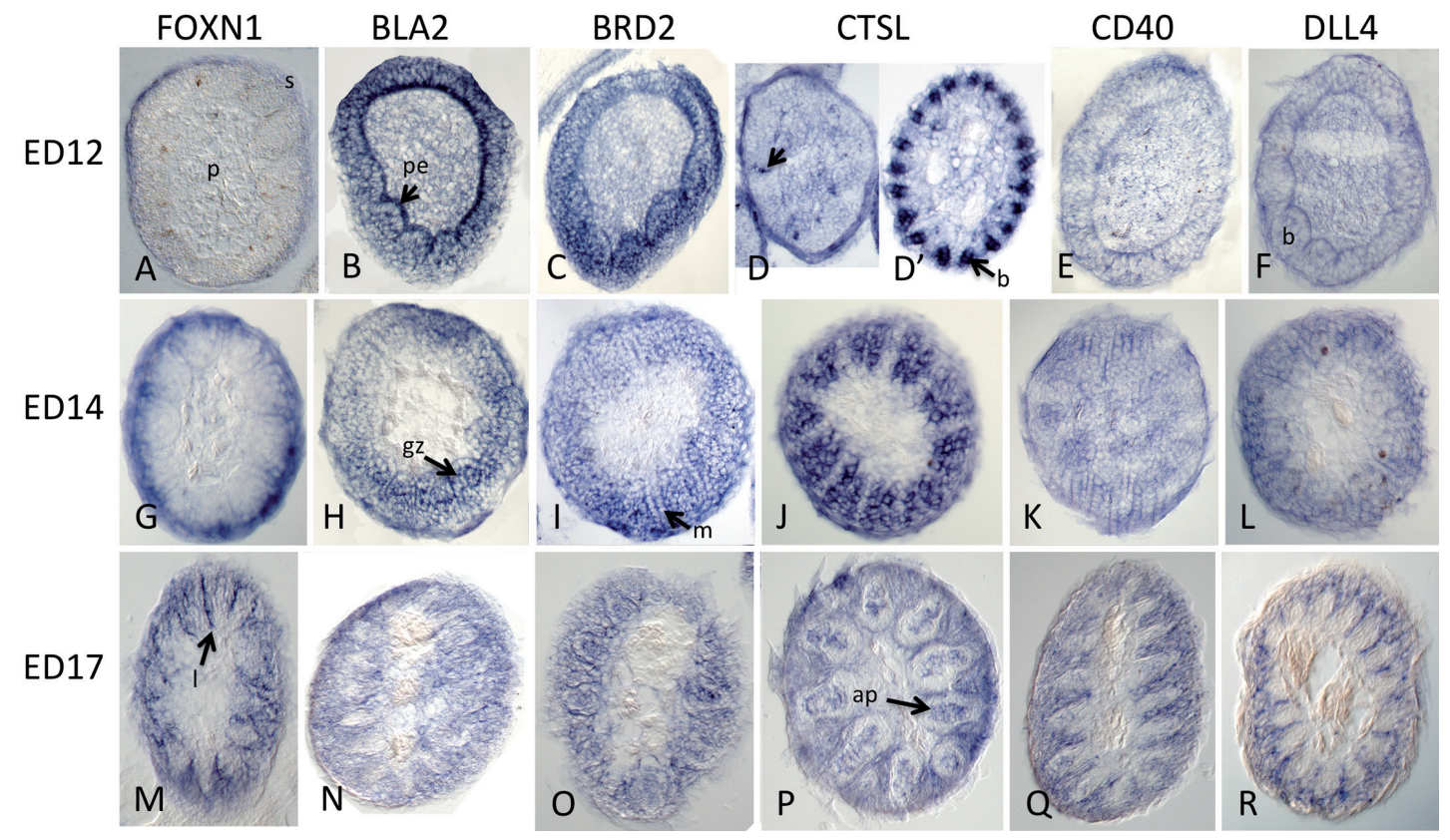

Fig. 4. Expression of FOXN1 and putative FOXN1 regulatory targets in embryonic day 12,14 and 17 feather cross sections. Photo columns are labeled with the gene name for the in situ probe, rows indicate the days of incubation. Panels show the results of in situ hybridization of probes for the genes FOXN1 $(\mathbf{A}, \mathbf{G}, \mathbf{M})$, BLA2 (B, H,N), BRD2 (C,I,O), CTSL (D,D',J,N), CD40 (E,KO) and DLL4 (F,L,R). FOXN1 and putative FOXN1 target genes show dynamic and interesting expression patterns in feather compartments. However, the expression of each of the genes is distinctly different from that of FOXN1 at similar stages and proximodistal levels. See text for detail. Structures are labeled in various panels to help with feather terminology: ap, axial plate (P); b, barb plates (F); gz, growth zone (H); I, line (M); m, marginal cells (I); p, pulp (A); pe, pulp epithelium (B); s, sheath (A).

skin whereas FOXN1 and BLA2 were not. Feather expression was similar but not identical between FOXN1 and the putative target genes for body feathers at ED12-17 (compare Fig. 3 rows ED12 skin, ED14 skin and ED17 skin). In ED14 feet (scales and nails) the difference in expression of these genes was more pronounced. FOXN1 mRNA was expressed in nails but not in scales (Fig. 3E', E"). BLA2 and BRD2 were expressed in scales not nails (Fig. 3F', F", G', G"). CTSL was expressed in scales and nails (Fig. $3 \mathrm{H}^{\prime}$, H"), whereas CD40 (Fig. 3 I', I") and DLL4 (Fig. 3 J', J") were expressed in neither scales nor nails. FOXN1 and CTSL expression were similar in nails. We conclude that all of the tested candidate target genes for FOXN1 are expressed in some avian epidermal appendages; however, direct FOXN1 regulation is not sufficient to explain all of their expression patterns.

To investigate specific localization of FOXN1 mRNA and these five candidate genes within feather filaments, hybridized feathers were paraffin sectioned transversely. At ED12 at the base of the feather where barb plates were just beginning to form, FOXN1 transcripts were predominantly localized in the feather sheath (Fig.4A), and more distally in the ends of the marginal plates (not shown) whereas transcripts of the five FOXN1 candidate targets did not share this pattern (Fig.4 B-F). For example, at ED12 BLA2 (and somewhat less so BRD2) mRNA was expressed in the pulp epithelium and within proximal barb plates. CTSL mRNA was ubiquitously expressed with stronger intensity in the sheath and distributed cells (Fig. 4D, arrow), whereas slightly more distally strong expression was uniquely seen in the barbule plates outside the growth zone (Fig. 4D'), a pattern not shared with any of the other probes. DLL4 and CD40 mRNA were weakly expressed in the outside layer of the feather sheath and in the pulp epithelium (Fig. 4 E,F). At ED13-14, in a more distal part of the filament, the overlap in expression patterns was more similar but with identifiable differences (Fig. 2L, 4G-L). FOXN1 was still expressed in the sheath, and also in the lateral barb plates and marginal cells.
Labeling of $B L A 2$ appeared stronger in the barb ridge (Fig. $4 \mathrm{H}$ ), $B R D 2$ labeled lateral barb plates, medial barb ridges and marginal cells equally (Fig. 4I) whereas CTSL was strongly expressed in the barb plates (Fig. 4J). Expression of CD40 and DLL4 persisted fairly ubiquitously at this level (Fig. $4 \mathrm{~K}, \mathrm{~L})$ with the exception of the dermal pulp for DLL4. By ED17 the strongest FOXN1 label was in a thin line of unknown fate between the barbs, with barb and marginal cells labeling but no label in sheath, medullary cells of the axial plate or pulp (Fig. 4M). A similar labeling pattern was observed for the other genes with the exception of the inter-marginal line, and that BRD2 and CTSL also labeled in the axial plate of the barbs (Fig. $4 \mathrm{~N}, \mathrm{R}$ ). Based on these data, the putative FOXN1 target genes show dynamic and interesting expression patterns in feather compartments. However, the expression of each of the genes is distinctly different from that of FOXN1 at similar stages and proximodistal levels.

\section{Discussion}

In the present study, we confirm that chick FOXN1 is expressed in the developing thymus and demonstrate its expression in some epidermal appendages, suggesting a homologous, evolutionarily conserved role for FOXN1 across amniotes. We have identified a novel expression pattern of FOXN1 in embryonic feathers. Chick FOXN1 initially is expressed along the length of the feather filament, with the exception of the base and tip. As the filament elongates between ED 12 and 17, FOXN1 expression becomes restricted to the proximal end of the feather. Although others have published differences in proximal-distal gene expression (e.g., Yu et al., 2002, Chodankar et al., 2003), to our knowledge FOXN1 and FOXE1 (Yaklichkin et al., 2011), the two FOXgenes we have characterized are the only known differentially and dynamically expressed genes along the proximal-distal axis of the elongating feather filament.

The murine ortholog of FOXN1 regulates expression of specific 
alpha keratins (Mecklenburg etal., 2004). Although hair forms from soft alpha-keratins and feathers form predominantly from hard beta-keratins, it is possible FOXN1 could be modulating expression of feather-specific keratins. Feather-specific beta-keratin expression coincides with differentiation of the barb ridges (Haake et al., 1984, König and Sawyer 1985). Images of feather filaments in the Haake paper indicate the proximal to distal pattern of beta-keratin expression correlates well with the expression pattern of FOXN1 shown here (Fig. 2). Specifically, beta-keratin expression is initiated at stage 38 (ED12) and expressed in the ring sheath cells similar to our image in Fig. $2 \mathrm{~K}$, whereas more distal expression is similar to Fig. 2L. Patterns of alpha-keratins also correlate with FOXN1 expression (Alibardi, 2013). Based on these observations and the expression of both mouse and chick FOXN1 in the nails and thymus, other keratinized epidermal structures, chick FOXN1 may regulate expression of both alpha and beta-keratins.

Finally, expression of FOXN1 and the other genes investigated in the feather raises more questions than it answers about the evolution of FOXN1 function, considering that the feather is thought to have evolved from scutate scales of reptiles. Loss and gain of function studies will be required to establish a precise role for FOXN1 transcriptional regulation in epidermal evolution and development.

\section{Materials and Methods}

\section{Sequence, data and phylogenetic analysis}

The protein sequences used in the analysis were obtained from the NCBI database (ncbi.nlm.nih.gov) and Ensemble database 69 (ensemble. org). The accession numbers of FOXN1/N4 protein sequences analyzed are provided in Supplementary Table S1. Multiple sequence alignments were constructed using T-COFFEE, version 7.7.1. (tcoffee.vital-it.ch/cgibin/Tcoffee/tcoffee_cgi/index.cgi). Aligned FOXN1/N4 sequences were edited using the alignment editor BioEdit 7.0.4.1. (mbio.ncsu.edu/bioedit/ page2.html). A phylogenic tree for FOXN1/N4 proteins was generated based on the forkhead DNA-binding domain sequences ( $\sim 100$ residues). A neighbor-joining method was used to construct the tree topology. The phylogenetic tree was converted into a cladogram using MEGA 4 (Kumar et al., 2004) (metameme.sdsc.edu).

\section{Incubation and isolation of chicken tissues}

Fertilized white leghorn eggs (Gallus gallus) were incubated in a forceddraft incubator at approximately $38^{\circ} \mathrm{C}$ with high humidity. Slight variations in temperature between incubators or over the course of the experiment make staging approximate due to long incubation times, therefore days of incubation are given. For older embryos, neck/thorax and skin/feathers were isolated prior to fixation with paraformaldehyde.

\section{Molecular cloning and in situ hybridization with gene probes}

To clone chicken FOXN1 cDNAsequences for the in situ probe template, mRNA was isolated from day 5 chicken embryos using the Qiagen RNeasy Mini Kit (cat. number: 74104) and cDNA synthesized using standard procedures. Template for probe generation was generated by PCR with the GC-rich PCR System Kit (cat. number: 12140306 001), Roche Applied Science; 34 cycles of annealing at $50^{\circ} \mathrm{C}$ and the following primers: forward primer, 5'-TTATAAAAGCTTGCGGCCGCAGAATATCACTACCCCTACCAAAGGATTGC-3'; reverse primer: 5'-GCTCTAGAAATTAACCCTCACTAAAGGGCCTCCCGCCTCCCCAGAAGGAAACATTG-3'. Chick FOXN1 sequences are in bold, the T3 RNA polymerase binding site is italicized. The PCR generated template was sequenced to confirm identity (NCBI Accession number: XM_415816.3, 904-1622 bp). A 719 nt RNA probe of FOXN1 was produced using T3 polymerase and in situ hybridizations were performed using the GEISHA mRNA Detection Protocol (geisha.arizona. edu/geisha/protocols.jsp). FOXE1 in situ hybridization was performed as previously described (Yaklichkin et al., 2011). Probe for MHC Class II gene, BLA2 (NCBI Acc. \#: NM_001245061) was generated from GEISHA plasmid W34 with Not I using T7 polymerase. Probe for BRD2 was generated from plasmid ChEST826a15 (NM_001030674.1) with Notl and T3 polymerase. Probe for Cathepsin L, CTSL2 (NM_001168009) was generated from GEISHA plasmid S12 with Notl and T7 polymerase. Probe for CD40 (TNFRSF5, NM_204665) was generated by PCR: Forward primer: 5'- CTGACAAGCAGTATGAGCACAAGG -3', Reverse primer: 5'-ATTAACCCTCACTAAAGGGGAAGTCCACCTCCTCGGG-3', where the bold sequence allows for directional amplification of the $666 \mathrm{nt}$ probe using the T3 polymerase. Probe DLL4 (XM_421132) was generated from plasmid pgf2n.pk001.e7 (www.chickest.udel.edu) using Notl and T3 polymerase. Probe sequences and additional images are available at http://geisha. arizona.edu/geisha.

\section{Aknowledgments}

We are thankful to Tatiana Yatskievych and Terry Sesepasara for technical assistance. This work was supported by NIH grant GM085226 and NIH NICHD grant P41HD064559.

\section{References}

ALIBARDI L (2009). Cornification of the pulp epithelium and formation of pulp cups in downfeathers and regenerating feathers. Anat Sci Int. 84: 269-279.

ALIBARDI L (2013) Immunolocalization of alpha-keratins and feather beta-proteins in feather cells and comparison with the general process of cornification of the skin of mammals. Ann Anat 195: 189-198.

BRANCACCIO A, MINICHIELLO A, GRACHTCHOUK M, ANTONINI D, SHENG H, PARLATO R, DATHAN N, DLUGOSZ AA, MISSERO C (2004). Requirement of the forkhead gene Foxe1, a target of sonic hedgehog signaling, in hair follicle morphogenesis. Hum Mol Genet 13: 2595-2606.

CHODANKAR R, CHANG CH, YUE Z, JIANG TX, SUKSAWEANG S, BURRUS LW, CHUONG CM AND WIDELITZ RB (2003). Shift of Localized Growth Zones Contributes to Skin Appendage Morphogenesis: Role of the Wnt/b-catenin Pathway. J. Invest Dermatol 120: 20-26.

ECKHART L, VALLE LD, JAEGER K, BALLAUN C, SZABO S, NARDI A, BUCHBERGER M, HERMANN M, ALIBARDI L, TSCHACHLER E (2008). Identification of reptilian genes encoding hair keratin-like proteins suggests a new scenario for the evolutionary origin of hair. Proc Natl Acad Sci USA 105: 18419-18423.

FRANK J, PIGNATA C, PANTELEYEV AA, PROWSE DM, BADEN H, WEINER L, GAETANIELLO L, AHMAD W, POZZI N, CSERHALMI-FRIEDMAN PB, AITA VM, UYTTENDAELE H, GORDON D, OTT J, BRISSETTE JL, CHRISTIANO AM (1999). Exposing the human nude phenotype. Nature 398: 473-474.

FURLONG RF (2005). Insights into vertebrate evolution from the chicken genome sequence. Gen Biol 6: 207

GLENN TC, FRENCH JO, HEINCELMAN TJ, JONES KL, SAWYER RH (2008) Evolutionary relationships among copies of feather beta (\{beta\}) keratin genes from several avian orders. Integr Comp Biol 48: 463-475.

HAAKEAR, KÖNIG G, SAWYERRH (1984). Avian feather development: relationships between morphogenesis and keratinization. Dev Biol 106: 406-413.

hESNet: Human Embryonic Stem Cell Transcription Network www.wanglab.ucsd edu/star/hESnet/gene.jsp?gene=FOXN1; accessed Dec. 4, 2013.

KAESTNER KH, KNOCHEL W, MARTINEZ DE (2000). Unified nomenclature for the winged helix/forkhead transcription factors. Genes Dev 14: 142-146.

KÖNIG G, SAWYER RH (1985). Analysis of morphogenesis and keratinization in transfilter recombinants of feather-forming skin. Dev Biol 109: 381-392.

KUMAR S, HEDGES SB (1998). A molecular timescale for vertebrate evolution Nature 392: 917-920.

KUMAR S, TAMURA K, NEI M (2004). MEGA3: Integrated software for Molecular Evolutionary Genetics Analysis and sequence alignment. Brief Bioinform 5: 150-163.

LEE D, PROWSE DM, BRISSETTE JL. (1999) Association between mouse nude gene expression and the initiation of epithelial terminal differentiation. Dev Biol. 208: 362-374.

LEE J, TUMBAR T (2009). Hairy tale of signaling in hair follicle development and 


\section{D.K. Darnell et al.}

cycling. Semin Cell Dev Biol 23: 906-916.

LEE YH, WILLIAMS A, HONG CS, YOU Y, SENOO M, SAINT-JEANNET JP (2013). Early development of the thymus in Xenopus laevis. Dev Dyn 242: 164-178.

LIN KK, KUMAR V, GEYFMAN M, CHUDOVA D, IHLER AT, SMYTH P, PAUS R, TAKAHASHI JS, ANDERSEN B (2009). Circadian Clock Genes Contribute to the Regulation of Hair Follicle Cycling. PLoS Genet 5: e1000573.

MA D, WANG L, WANG S, GAO Y, WEI Y, LIU F (2012). FOXN1 maintains thymic epithelial cells to support T-cell development via $\mathrm{mcm} 2$ in zebrafish. Proc Natl Acad Sci USA 109: 21040-21045.

MECKLENBURG L, NAKAMURA M, SUNDBERG JP, PAUS R (2001). The nude mouse skin phenotype: the role of FOXN1 in hair follicle development and cycling. Exp Mol Pathol 71: 171-178.

MECKLENBURG L, PAUS R, HALATAZ, BECHTOLDLS, FLECKMANP, SUNDBERG JP (2004). FOXN1 is critical for onycholemmal terminal differentiation in nude (FOXN1) mice. J Invest Dermatol 123: 1001-1011.

MECKLENBURG L, TYCHSEN B, PAUS R (2005). Learning from nudity: lessons from the nude phenotype. Exp Dermatol 11: 797-810.

MEIER N, DEAR TN, BOEHM T (1999). Whn and mHa3 are components of the genetic hierarchy controlling hair follicle differentiation. Mech Dev 89: 215-221.

NEVES H, DUPIN E, PARREIRA L, LE DOUARIN NM (2012). Modulation of Bmp4 signalling in the epithelial-mesenchymal interactions that take place in early thymus and parathyroid development in avian embryos. Dev Bio/361: 208-219.
NOWELL CS, BREDENKAMP N, TETÉLIN S, JIN X, TISCHNER C, VAIDYA H SHERIDAN JM, STENHOUSE FH, HEUSSEN R, SMITH AJ, BLACKBURN CC (2011). FOXN1 regulates lineage progression in cortical and medullary thymic epithelial cells but is dispensable for medullary sublineage divergence. PLOS Genet 7:e1002348.

PIGNATA C, FUSCO A, AMOROSI S (2009). Human clinical phenotype associated with FOXN1 mutations. Adv Exp Med Biol 665: 195-206

RODEWALD HR (2008) Thymus Organogenesis. Annu Rev Immunol 26: 355-388.

SCHLAKET, SCHORPPM, MAUL-PAVICICA, MALASHENKO AM, BOEHMT (2000). Forkhead/winged-helix transcription factor Whn regulates hair keratin gene expression: molecular analysis of the nude skin phenotype. Dev Dyn 217: 368-376.

SCHÜDDEKOPF K, SCHORPP M, BOEHM T (1996). The whn transcription factor encoded by the nude locus contains an evolutionarily conserved and functionally indispensable activation domain. Proc Natl Acad Sci USA. 93: 9661-9664.

SEGRE JA, NEMHAUSER JL, TAYLOR BA, NADEAU JH, LANDER ES (1995). Positional cloning of the nude locus: genetic, physical, and transcription maps of the region and mutations in the mouse and rat. Genomics 28: 549-559.

YAKLICHKIN SY, DARNELL DK, PIER MV, ANTIN PB, HANNENHALLI S (2011). Accelerated evolution of 3'avian FOXE1 genes, and thyroid and feather specific expression of chicken FoxE1. BMC Evol Biol 11: 302.

YU M, WU P, WIDELITZ RB, AND CHUONG C-M (2002) The morphogenesis of feathers. Nature 420: 308-312.

YU M, YUE Z, WU P, WU DY, MAYER JA, MEDINA M, WIDELITZ RB, JIANG TX, CHUONG CM (2004) The biology of feather follicles. Int J Dev Biol 48: 181-191. 


\section{Further Related Reading, published previously in the Int. J. Dev. Biol.}

Regulatory $\mathrm{T}$ cells in the establishment and maintenance of self-tolerance: role of the thymic epithelium Josselyne Salaün, Catherine Corbel and Nicole M. Le Douarin

Int. J. Dev. Biol. (2005) 49: 137-142

http://dx.doi.org/10.1387/ijdb.041959js

How and when the regional competence of chick epidermis is established: feathers vs. scutate and reticulate scales, a problem en route to a solution

Fabrice Prin and Danielle Dhouailly

Int. J. Dev. Biol. (2004) 48: 137-148

http://dx.doi.org/10.1387/ijdb.15272378

Integument pattern formation involves genetic and epigenetic controls: feather arrays simulated by digital hormone models

Ting-Xin Jiang, Randall B Widelitz, Wei-Min Shen, Peter Will, Da-Yu Wu, Chih-Min Lin, HanSung Jung and Cheng-Ming Chuong

Int. J. Dev. Biol. (2004) 48: 117-135

http://dx.doi.org/10.1387/ijdb.15272377

Chick-embryo culture techniques employed at Karnatak University in Dharwad, India, for studying cellular and molecular aspects of morphogenesis Sohan P Modak

Int. J. Dev. Biol. (2003) 47: 165-170

http://dx.doi.org/10.1387/ijdb.12705665

\section{Early chick embryos in vitro}

C D Stern and R Bachvarova

Int. J. Dev. Biol. (1997) 41: 379-387

http://dx.doi.org/10.1387/ijdb.9184348

5 yr ISI Impact Factor $(2011)=2.959$
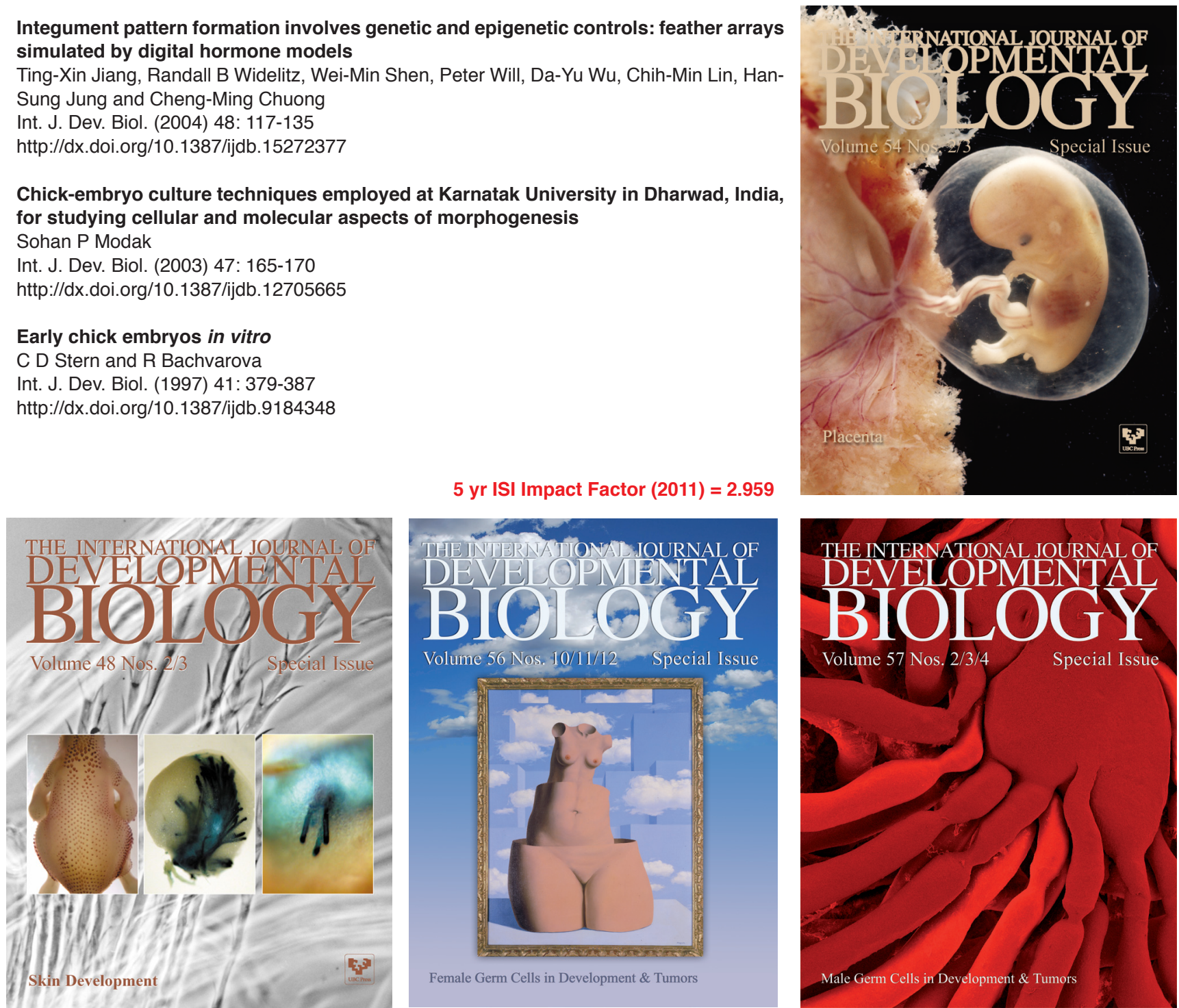